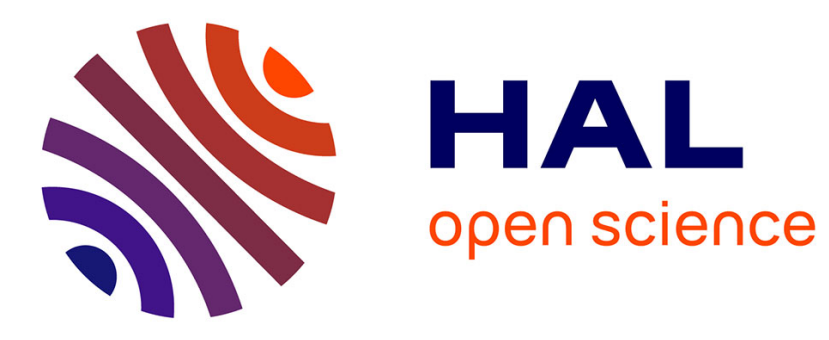

\title{
Incremental Cross-Modality Deep Learning for Pedestrian Recognition
}

\author{
Danut Ovidiu Pop, Alexandrina Rogozan, Fawzi Nashashibi, Abdelaziz \\ Bensrhair
}

\section{- To cite this version:}

Danut Ovidiu Pop, Alexandrina Rogozan, Fawzi Nashashibi, Abdelaziz Bensrhair. Incremental CrossModality Deep Learning for Pedestrian Recognition. IV'17 - IEEE Intelligent Vehicles Symposium , Jun 2017, Redondo Beach, CA, United States. 10.1109/IVS.2017.7995771 . hal-01501711v3

\section{HAL Id: hal-01501711 \\ https://hal.inria.fr/hal-01501711v3}

Submitted on 8 Jun 2017

HAL is a multi-disciplinary open access archive for the deposit and dissemination of scientific research documents, whether they are published or not. The documents may come from teaching and research institutions in France or abroad, or from public or private research centers.
L'archive ouverte pluridisciplinaire HAL, est destinée au dépôt et à la diffusion de documents scientifiques de niveau recherche, publiés ou non, émanant des établissements d'enseignement et de recherche français ou étrangers, des laboratoires publics ou privés. 


\title{
Incremental Cross-Modality Deep Learning for Pedestrian Recognition
}

\author{
Dănuţ Ovidiu Pop ${ }^{1}$, Alexandrina Rogozan ${ }^{2}$, Fawzi Nashashibi ${ }^{3}$ and Abdelaziz Bensrhair ${ }^{4}$
}

\begin{abstract}
In spite of the large number of existing methods, pedestrian detection remains an open challenge. In recent years, deep learning classification methods combined with multimodality images within different fusion schemes have achieved the best performance. It was proven that the late-fusion scheme outperforms both direct and intermediate integration of modalities for pedestrian recognition. Hence, in this paper, we focus on improving the late-fusion scheme for pedestrian classification on the Daimler stereo vision data set. Each image modality, Intensity, Depth and Flow, is classified by an independent Convolutional Neural Network (CNN), the outputs of which are then fused by a Multi-layer Perceptron (MLP) before the recognition decision. We propose different methods based on Cross-Modality deep learning of CNNs: (1) a correlated model where a unique CNN is trained with Intensity, Depth and Flow images for each frame, (2) an incremental model where a CNN is trained with the first modality images frames, then a second CNN, initialized by transfer learning on the first one is trained on the second modality images frames, and finally a third CNN initialized on the second one, is trained on the last modality images frames. The experiments show that the incremental cross-modality deep learning of CNNs improves classification performances not only for each independent modality classifier, but also for the multi-modality classifier based on late-fusion. Different learning algorithms are also investigated.
\end{abstract}

\section{INTRODUCTION}

Pedestrian detection is a challenging task of great importance in the domain of object recognition and computer vision. It is a key problem for surveillance, robotics applications and automotive safety [1] where an efficient Advanced Driver Assistance System (ADAS) for pedestrian detection is needed to reduce the number of accidents and fatal injures ${ }^{1}$. These systems usually have multi-modality sensors and/or camera networks to capture the road data, and signal/image processing components to extract pertinent features which are then classified by recognition components.

A study performed by ABI Research published in 2015 shows that Mercedes-Benz, Volvo and BMW dominate the market for car enhancing ADAS systems. As from 2013,

\footnotetext{
${ }^{1}$ Dănuţ Ovidiu Pop is a PhD student at RITS Team, INRIA Paris, 2 Rue Simone IFF, 75012 Paris, France in collaboration with Normandie Univ, INSA Rouen, LITIS, 76000 Rouen, France and Department of Computer Science, Babeş-Bolyai University, 7- 9 Universitatii street, 400084 ClujNapoca, Romania. danut-ovidiu.pop@inria.fr

2 Dr. Alexandrina Rogozan is Associate Professor at Normandie Univ, INSA Rouen, LITIS, 76000 Rouen, France. alexandrina.rogozan@insa-rouen. fr

${ }^{3}$ Dr. Fawzi Nashashibi is the head of RITS Team at INRIA Paris, 2 Rue Simone IFF, 75012 Paris, France. fawzi.nashashibi@inria.fr

${ }^{4}$ Dr. Abdelaziz Bensrhair is Professor at Normandie Univ, INSA Rouen, LITIS, 76000 Rouen, France. abdelaziz.bensrhaireinsa-rouen. fr

${ }^{1}$ According to European Commission statistics published in 2016, the number of pedestrians injured in road accidents in 2014 was 1,419,800 and there were 25,900 fatalities
}

BMW cars have been fitted with a Driver Assistance package for Pedestrian Warning, based on infrared night-vision and monocular vision cameras. Recently, the Mercedes system has combined stereo vision cameras with long, medium and short-range radars to monitor the area in front of the vehicle. In 2016 the Continental company proposed an Advanced Radar Sensor (standard for VW Tiguan) able to detect both objects and pedestrians, at a distance of up to 170 meters. The Nissan company developed a system which detects the vehicle's environment, including as the road, other vehicles and pedestrians.

These existing ADAS systems still have difficulty distinguishing between human beings and nearby objects, especially in a crowded urban environment where they are not able to detect all partially occluded pedestrians, and they do not work efficiently in extreme weather conditions. Moreover, it is difficult to find an ADAS system that is able to ensure stable, real-time and effective full functionality. We believe it is necessary to improve the classification component of an ADAS system to be able to discriminate between the obstacle type (pedestrian, cyclist, child, old person) in order to adapt the car driver system behavior according to the estimated risk level.

Our work is concerned with improving the classification component of a pedestrian detector. In recent research studies, deep learning neural networks including convolutional neural networks (CNNs), like LeNet, AlexNet, GoogLeNet, have usually led to improvement in classification performance [2], [3], [4]. Moreover, deep learning classification methods combined with multi-modality images within different fusion schemes have achieved remarkable results. The disadvantage of those models is that they require a large amount of annotated data for each modality.

It usually happens that one has not (enough) annotated data in one modality compared with other modalities. The question is whether one modality can be used exclusively (standpoint one) for training the classification model used to recognize pedestrians in another modality or only partially (standpoint two) for improving the training of the classification model in another modality. To our knowledge, this question has not yet been answered for the pedestrian recognition task. This paper proposes to solve this brainteaser through various experiments based on the Daimler stereo vision data set [5].

The paper is organized as follows: Section 2 briefly presents our main contribution and some existing approaches from the literature. Section 3 presents the architecture and methods approach based on Cross-Modality deep learning of CNNs. Section 4 presents the experiments and their 
results on the Daimler dataset. Finally, Section 5 presents our conclusions.

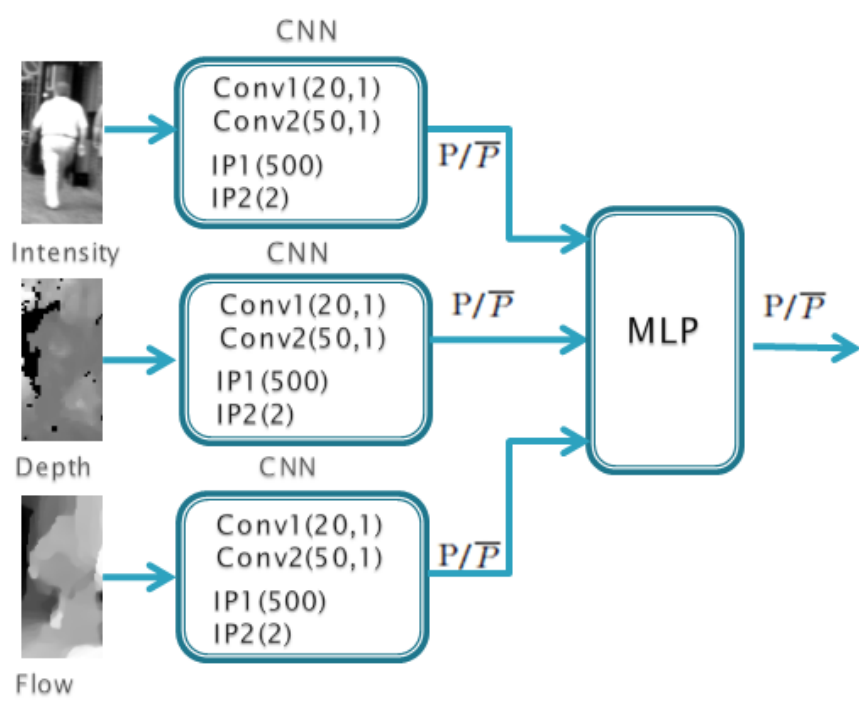

Fig. 1. The correlated cross-modality training architecture

\section{PREVIOUS WORK}

Over the last decade, the pedestrian detection issue has been investigated intensely, resulting in the development of detection methods using a combination of features such as Integral Channel Features [6], Histograms of Oriented Gradients (HOG) [7], Local Binary Patterns (LBP), Scale Invariant Feature Transform (SIFT) [8], among others, followed by a trainable classifier such as a Support Vector Machine (SVM), Multilayer Perceptrons (MLP), boosted classifiers or random forests [9], [10]. In [11] the authors present a mixtureof-experts framework performed with HOG, LBP features and MLP or linear SVM classifiers. Recently, in [12] a $\mathrm{CNN}$ to learn the features with an end-to-end approach was presented. This experiment focused on the detection of small scale pedestrians on the Caltech data set. A combination of three CNNs to detect pedestrians at different scales was proposed on the same monocular vision data set [13]. A cascade Aggregated Channel Features detector is used in [14] to generate candidate pedestrian windows followed by a CNN-based classifier for verification purposes on monocular Caltech and stereo ETH data sets. Two CNN-based fusion methods of visible and thermal images on the KAIST multispectral pedestrian data set were presented in [15]. The first method combines the information of these modalities at the pixel level (early fusion), while the second architecture uses separate sub-networks to generate a feature representation for each modality before classification (intermediate fusion). The authors showed that the intermediate fusion outperforms the early fusion.

We compared in [16] the performance of the early fusion and late fusion models on the Daimler stereo vision data set. The early fusion model was built by concatenating three image modalities (intensity, depth and flow) to feed a unique $\mathrm{CNN}$. The late fusion model consists in fusing the outputs of three independent CNNs, trained on intensity, depth and flow images, by an SVM classifier. We showed the early-fusion model is less efficient than the late-fusion model. Moreover, the early fusion is less robust than the late fusion, since it needs strong image calibration and synchronization. Its training is less effective since for a given image frame it needs an item for each modality and therefore the classifier requires more items to learn the problem. With the earlyfusion model, it is impossible to take advantage of interdataset training methods by using modality images from different unimodal and/or multi-modal datasets where all the modalities are not acquired and/or annotated, in order to improve the training by extending the number and the variety of items.

In the literature, in studies on intermediate and late fusion methods, the training was made independently on each modality, exclusively with annotated images acquired from that modality. The aim of this paper is to improve the latefusion training by using a cross-modality approach. We will prove that the incremental cross-modality is effective for the training of each modality classifier not only with images from that modality, but also with images from other modalities: Intensity, Depth and Flow. A synthetic dataset (Virtual Pedestrian dataset [17]) is used for an initial training, and two different real-world datasets (KITTI Vision Benchmark Suite and the Daimler Mono Pedestrian Detection Benchmark) for fine-tuning and evaluation.

To the best of our knowledge, no study has been carried out on cross-modality training for pedestrian recognition. In [18], the authors proposed an incremental cross-dataset learning algorithm for the pedestrian detection problem.

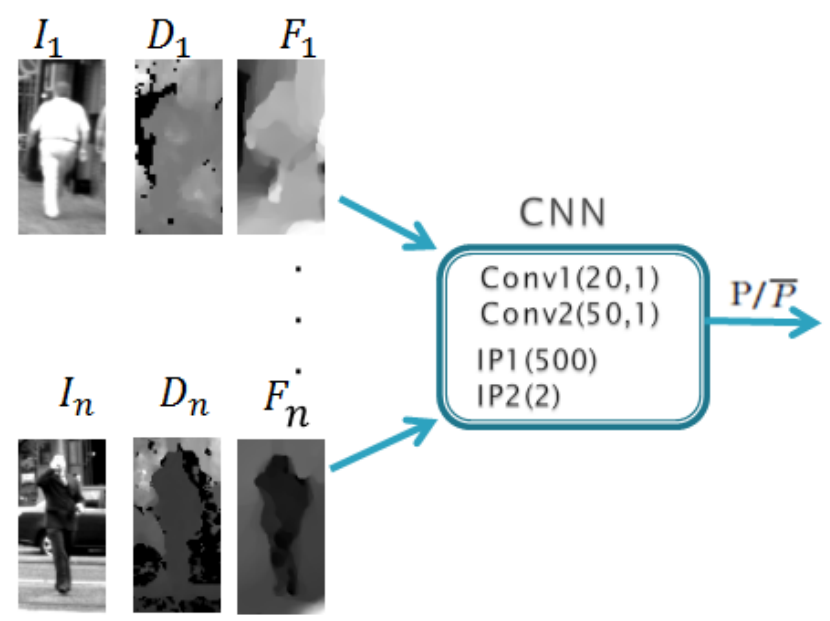

Fig. 2. The general CNN architecture for input data set inserted consecutively, intensity, depth and flow, which trains and validates on the identical multi-modal or unimodal data.

\section{THE PROPOSED ARCHITECTURES}

In this paper, we propose fusing stereo-vision information between three modalities: Intensity (I), Depth (D) and Flow (F). We investigate the late-fusion architecture using three 


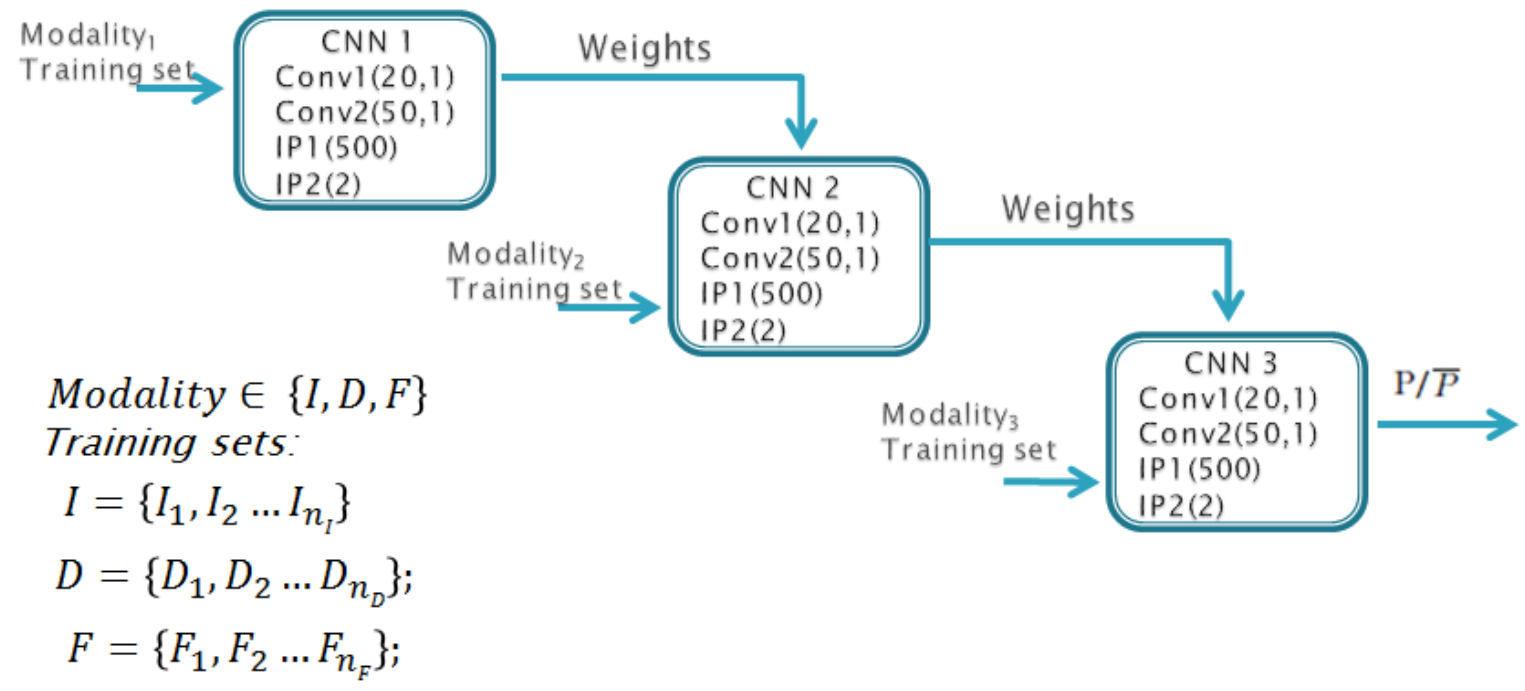

Fig. 3. The incremental cross-modality deep learning architecture.

different methods for the training of the CNN-based classifiers: a classical intra-modality approach and two different methods for a cross-modality approach.

\section{A. Our baseline late fusion architecture}

We propose a late-fusion architecture (see Fig 1) where an MLP is used to discriminate between pedestrians (P) and non-pedestrians $(\bar{P})$ on the classification results (the class probability estimate of the previous estimated class) of three modality independent CNNs. Each CNN is exclusively trained on the same modality in an independent manner: trained and validated on the same modality.

Each modality CNN is based on the LeNet architecture which consists of 7 layers, apart from the input layer, 2 convolutional layers, 2 pooling layers, 2 inner product (IP) layers and one rectified linear unit (ReLU) layer. We use 20 filters with one stride for the first convolutional layer followed by 50 filters with one stride for the second one. We use two IP layers with 500 neurons for the first IP layer and 2 neurons for the second IP layer. The final layer returns the final decision of the classifier system: $\mathrm{P}$ or $\bar{P}$.

\section{B. Correlated cross-modality training of CNNs}

We propose a correlated cross-modality approach where a unique CNN is learned with the same image frames, but provided in different modalities, Intensity $I_{i}$, Depth $D_{i}$ and Flow $F_{i}$ with $\mathrm{i}=1$,n (see Fig. 2). The CNN model is validated in two different ways: on a multi-modality validation set and on a single modality one. The learning and validation sets are disjointed.

We believe that the drawback of correlated cross-modality training is that it compels one to use a unique CNN model. This is too strong a constraint, if different modalities can improve the learning process with different $\mathrm{CNN}$ architectures and/or with different settings (learning algorithms and learning rates).

\section{Incremental cross-modality training}

Our experiments show that not all modality CNNs provide the best results with the different architectures and settings. Therefore, we propose an incremental cross-modality training, based on a transfer learning approach (see Fig.3).

A first CNN is trained and validated with the first modality images frames, then a second CNN, initialized by transfer learning on the first $\mathrm{CNN}$, is trained and validated on the second modality image frames, and finally a third CNN initialized on the second $\mathrm{CNN}$, is trained and validated on the last modality images frames. Transferring learning consists in transferring weighted information from a previous $\mathrm{CNN}$ that has already been learned, to a new $\mathrm{CNN}$ which is to be trained.

The advantage of this method is that its architecture is more flexible allowing for adaptive settings for each classifier (i.e. different learning algorithms and rate policies). Learning this model does not require any inter-modality correlated data, nor synchronized modality images. This could be an interesting point if the multi-modality images are various and not acquired with similar sensors/cameras and/or extracted from the same database. The approach can be extended to cross-datasets training.

For this approach, the question is whether the order of modality training within the previous model has any importance. We investigate different combinations and we conclude that for the classification in the Intensity modality, the optimal order for training is Depth images first, followed by Flow images and finally Intensity images (D,F,I training model of I); for the classification in the Flow modality the optimal order for training is Depth images first, followed by Intensity images and finally Flow images (D,I,F training model of F), and respectively for the classification in the Depth modality the optimal order for training is Intensity images first, followed by Flow images and finally Depth images (I,F,S for training of D) (see Table II). 
TABLE I

COMPARISON OF LEARNING ALGORITHMS AND RATE POLICIES ON INTENSITY, DEPTH AND FLOW DATA SET

\begin{tabular}{|c|c|c|c|c|c|c|c|c|}
\cline { 5 - 9 } \multicolumn{2}{c|}{} & \multicolumn{7}{|c|}{ Accuracy } \\
\hline \multirow{3}{*}{ Modality Type } & Learning rate polics & \multirow{2}{*}{ EXP } & FIX & INV & POLY & SIG & \multirow{2}{*}{ STEP } & \multirow{2}{*}{ MS } \\
\cline { 2 - 9 } & Algorithm Learning & & & & & & & \\
& SGD & $95.96 \%$ & $96.07 \%$ & $96.01 \%$ & $96.09 \%$ & $96.01 \%$ & $96.20 \%$ & $95.78 \%$ \\
& RMSPROP & $95.53 \%$ & $61.19 \%$ & $95.24 \%$ & $\mathbf{9 6 . 5 5 \%}$ & $96.42 \%$ & $95.91 \%$ & $93.37 \%$ \\
& ADADELTA & $88.67 \%$ & $93.08 \%$ & $91.77 \%$ & $88.79 \%$ & $91.96 \%$ & $91.10 \%$ & $89.75 \%$ \\
& ADAGRAD & $95.02 \%$ & $95.41 \%$ & $95.83 \%$ & $95.49 \%$ & $95.46 \%$ & $95.87 \%$ & $95.02 \%$ \\
\hline \multirow{5}{*}{ Depth } & SGD & $\mathbf{8 9 . 7 8 \%}$ & $61.2 \%$ & $89.26 \%$ & $89.69 \%$ & $88.24 \%$ & $88.97 \%$ & $61.2 \%$ \\
& RMSPROP & $88.64 \%$ & $61.17 \%$ & $81.99 \%$ & $89.10 \%$ & $88.66 \%$ & $89.22 \%$ & $83.54 \%$ \\
& ADADELTA & $87.14 \%$ & $88.11 \%$ & $87.64 \%$ & $87.27 \%$ & $88.24 \%$ & $87.72 \%$ & $87.77 \%$ \\
& ADAGRAD & $88.77 \%$ & $88.81 \%$ & $89.44 \%$ & $89.25 \%$ & $89.44 \%$ & $89.09 \%$ & $88.71 \%$ \\
\hline \multirow{5}{*}{ Flow } & SGD & $86.53 \%$ & $61.2 \%$ & $86.69 \%$ & $86.90 \%$ & $86.72 \%$ & $86.84 \%$ & $61.2 \%$ \\
& RMSPROP & $86.89 \%$ & $61.91 \%$ & $80.33 \%$ & $85.69 \%$ & $87.16 \%$ & $86.33 \%$ & $86.57 \%$ \\
& ADADELTA & $86.56 \%$ & $\mathbf{8 7 . 3 4 \%}$ & $87.08 \%$ & $86.78 \%$ & $87.03 \%$ & $86.82 \%$ & $87.18 \%$ \\
& ADAGRAD & $87.22 \%$ & $86.46 \%$ & $87.11 \%$ & $86.17 \%$ & $86.59 \%$ & $86.68 \%$ & $86.97 \%$ \\
\hline
\end{tabular}

TABLE II

PERFORMANCE OF INCREMENTAL VS CROSS-MODALITY CLASSIFIERS

\begin{tabular}{|c|c|c|c|c|c|}
\hline Trained on & Validated on & Tested on & TPR & FPR & ACC \\
\hline Correlated cross-modality & Intensity & Intensity & 0.972 & 0.0737 & $94.4 \%$ \\
Correlated cross-modality & Depth & Depth & 0.9112 & 0.0172 & $86.06 \%$ \\
Correlated cross-modality & Flow & Flow & 0.9115 & 0.152 & $87.38 \%$ \\
\hline Depth+Flow+Intensity & Depth, Flow, Intensity & Intensity & 0.9619 & 0.029 & $96.7 \%$ \\
Intensity+Flow+Depth & Intensity, Flow, Depth & Depth & 0.8764 & 0.095 & $89.39 \%$ \\
Depth+Intensity+Flow & Depth, Intensity, Flow & Flow & 0.9436 & 0.056 & $94.34 \%$ \\
\hline
\end{tabular}

\section{EXPERIMENTS AND RESULTS}

The training and testing were carried out on Daimler stereo vision images of 48 x 96 px with a 12-pixel border around the pedestrian images extracted from three modalities: Intensity, Depth and optical Flow.

We use 84577 samples for training, $75 \%$ of which are used for learning, $25 \%$ for validation and 41834 for testing. The training set contains:

- 52112 samples of pedestrians

- 32465 samples of non pedestrians

The testing set contains:

- 25608 samples of pedestrians

- 16235 samples of non pedestrians

The experiments are performed in the Caffe deep neural network framework. The performances are measured by the Accuracy (ACC) and using the Receiver Operating Characteristics (ROC) curve created by plotting the true positive rate (TPR) against the false positive rate (FPR) at various threshold settings. The complexity of the classification system is also investigated by the area under the curve (AUC).

\section{A. Benchmark of uni-modal classifiers}

In [16], we compared the performance between AlexNet and LeNet on the Caltech dataset, the best performance measured by AUC was achieved with the LeNet architecture. From the Caltech image dataset, we selected pedestrians bounding boxes (BB) of more than $50 \mathrm{px}$. and all BB were resized to quadratic size $(64 \times 64 \mathrm{px})$. We have noticed the LeNet achieved the better result on smaller image datasets. Hence, we started by comparing for each modality images the classification performances with LeNet architecture with different learning algorithms: Stochastic
Gradient Descent (SGD), Adaptive Gradient (ADAGRAD), RMSPROP, ADADELTA and learning rate polices: Fixed (FIX), Exponential (EXP), Step Down (STEP), Polynomial Decay (POLY), Sigmoid (SIG), Multi-Step (MS) and Inverse Decay (INV) (see Table I). It is to be noted that the CNNs were optimized on the training set through 29760 epochs and 0.01 learning rate for both the single modality and the incremental cross-modality models. Each modality classifier is exclusively trained with imagines of its own modality. For the Intensity modality the best performance (ACC = 96.55\%) was achieved with the LeNet architecture using the RMSPROP $^{2}$ algorithm learning, with POLY rate policy. The best performances are obtained in Depth images with SGD and EXP settings ( $\mathrm{ACC}=89.78 \%)$ and respectively in Flow images ADADELTA and FIX settings $($ ACC $=87.34 \%)$. Therefore, different modalities need different learning algorithms and rate policies for an optimal training.

\section{B. Benchmark of cross-modality training methods}

The CNNs were optimized on the training set through 29760 epochs and 0.01 learning rate for both the single modality and the incremental cross-modality models. The CNN belonging to the correlated cross-modality approach needs three times more training epochs (89220 epochs) for the same learning rate.

Since the RMSPROP with POLY settings achieved the best performance on the Intensity modality, we decided to use the same settings to train the correlate cross-modality (CCM-CNN). The CNN model is validated following two

\footnotetext{
${ }^{2}$ Tieleman, T. and Hinton, G.,Lecture 6.5-RmsProp: Divide the gradient by a running average of its recent magnitude, COURSERA: Neural Networks for Machine Learning, 2012
} 
TABLE III

PERFORMANCE WITH LATE FUSION ON DAIMLER TESTING SET

\begin{tabular}{|l|l|l|l|}
\hline Late-fusion & TPR & FPR & ACC \\
\hline classical training & 0.9518 & 0.0109 & $97.46 \%$ \\
\hline Incremental cross modality & 0.9534 & 0.0092 & $\mathbf{9 7 . 6 2} \%$ \\
\hline
\end{tabular}

different approaches on the multi-modality union data set, and single modality ones (see Table II). The second approach gives better results. This correlated cross-modality training outperforms the classical intra-modality training only on the Flow testing set. This may be explained by the fact that, with more complex training data, the breadth and depth of the network should be increased. However the complexity would be limited by computing resources, which would thus hinder performance (see Table II) [19], [20].

For the training following the incremental cross-modality method, we use RMSPROP with POLY settings for all three CNNs through 29760 epochs. The results, given in Table II are better than those achieved with classical training (see Table I) for the same settings.

\section{Late-fusion with classical vs cross-modality training}

In Table III we show the performances obtained with classical training versus incremental cross-modality. The incremental cross-modality late-fusion solution we propose outperforms not only all the single modality classifiers but also the classical late-fusion solution. However, the incremental cross-modality is not statistically signified. These performances are also shown in the ROC curves (see Fig $4)$.

\section{CONCLUSIONS}

In this paper, we proposed different cross-modality training approaches for late-fusion architectures to improve pedestrian recognition. We have presented systematic experimental evidence on the effectiveness of deep neural networks for pedestrian recognition. We use LeNet architectures with RMSPROP algorithm learning for pedestrian recognition based on a multi-modal image data set. We also evaluated two CNN architectures, one for the incremental correlated method and the other for the correlated cross-modality method. The best performance was achieved with the late fusion architecture which used outputs from the incremental CNNs approaches. The best incremental combination was obtained with Depth, Flow, Intensity for Intensity method followed by Intensity, Flow and Depth for Depth method and Depth, Intensity, Flow for Flow method. The pedestrian recognition issue still remains an important challenge because its accuracy and robustness can be still improved.

The increase in the complexity of the issue requires increasing the breadth and depth of the network as it is limited by computing resources which hinders the performance. The incremental correlated cross-modality approach outperforms the correlated cross-modality approach as it improves the classification performance compared to a classical training of unimodal CNNs through late-fusion schemes on the Daimler data set. This method is more flexible allowing for adaptive

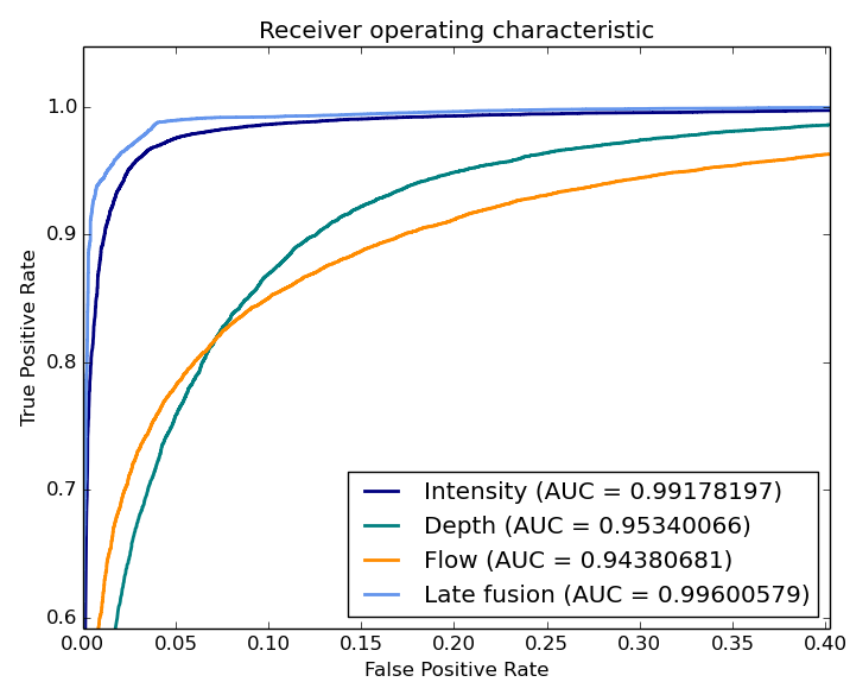

(a) Classical training

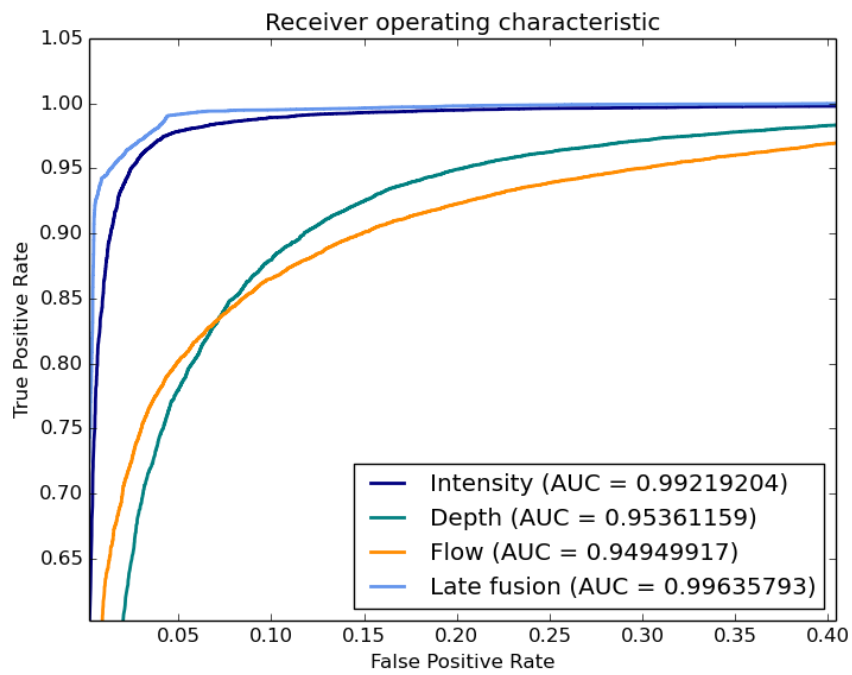

(b) Incremental cross-modality

Fig. 4. ROC classification performance on Daimler testing data set.

settings for each CNN classifier while the correlated crossmodality method compels to use a unique CNN model.

The late fusion methods achieves a better performance because it has a complex architecture which allows independent training of its components (it combines the output scores of all classifiers). The incremental cross-modality late-fusion outperforms all the single modality classifiers and classical late-fusion solutions but its performance is not statistically signified. We think this method could provide better results if it is applied on the suitable neural networks. We believe that the correlated approach is the most promising one.

For future work, we will improve our models with new CNN architecture by creating a new neural network, the performance of which will not be hindered by complex computing resources. We will concentrate on improving that model using optimal settings for different training modality 
sets and also by extending the model to cross-dataset training.

\section{ACKNOWLEDGEMENTS}

The research for this paper was financially supported by the Normandy Region and Inria Paris.

\section{REFERENCES}

[1] Pierre Sermanet, Koray Kavukcuoglu, Soumith Chintala, and Yann Lecun. Pedestrian detection with unsupervised multi-stage feature learning. In The IEEE Conference on Computer Vision and Pattern Recognition (CVPR), June 2013.

[2] Jan Hosang, Mohamed Omran, Rodrigo Benenson, and Bernt Schiele. Taking a deeper look at pedestrians. In IEEE Conference on Computer Vision and Pattern Recognition (CVPR), June 2015.

[3] H. Fukui, T. Yamashita, Y. Yamauchi, H. Fujiyoshi, and H. Murase. Pedestrian detection based on deep convolutional neural network with ensemble inference network. In 2015 IEEE Intelligent Vehicles Symposium (IV), pages 223-228, June 2015.

[4] Anelia Angelova, Alex Krizhevsky, and Vincent Vanhoucke. Pedestrian detection with a large-field-of-view deep network. In IEEE International Conference on Robotics and Automation, ICRA 2015, Seattle, WA, USA, 26-30 May, 2015, pages 704-711, 2015.

[5] M. Enzweiler, A. Eigenstetter, B. Schiele, and D. M. Gavrila. Multicue pedestrian classification with partial occlusion handling. In 2010 IEEE Computer Society Conference on Computer Vision and Pattern Recognition, pages 990-997, June 2010.

[6] Piotr Dollar, Zhuowen Tu, Pietro Perona, and Serge Belongie. Integral channel features. In Proc. BMVC, pages 91.1-91.11, 2009. doi:10.5244/C.23.91.

[7] Navneet Dalal and Bill Triggs. Histograms of oriented gradients for human detection. In Proceedings of the 2005 IEEE Computer Society Conference on Computer Vision and Pattern Recognition (CVPR'05) - Volume 1 - Volume 01, CVPR '05, pages 886-893, Washington, DC, USA, 2005. IEEE Computer Society.

[8] A. Vedaldi, V. Gulshan, M. Varma, and A. Zisserman. Multiple kernels for object detection. In Proceedings of the International Conference on Computer Vision (ICCV), 2009.

[9] Rodrigo Benenson, Mohamed Omran, Jan Hosang, and Bernt Schiele. Ten Years of Pedestrian Detection, What Have We Learned?, pages 613-627. Springer International Publishing, Cham, 2015.

[10] Piotr Dollar, Christian Wojek, Bernt Schiele, and Pietro Perona. Pedestrian detection: An evaluation of the state of the art. IEEE Trans. Pattern Anal. Mach. Intell., 34(4):743-761, April 2012.

[11] M. Enzweiler and D. M. Gavrila. A multilevel mixture-of-experts framework for pedestrian classification. IEEE Transactions on Image Processing, 20(10):2967-2979, Oct 2011.

[12] R. Bunel, F. Davoine, and Philippe Xu. Detection of pedestrians at far distance. In 2016 IEEE International Conference on Robotics and Automation (ICRA), pages 2326-2331, May 2016.

[13] M. Eisenbach, D. Seichter, T. Wengefeld, and H. M. Gross. Cooperative multi-scale convolutional neural networks for person detection. In 2016 International Joint Conference on Neural Networks (IJCNN), pages 267-276, July 2016.

[14] Xiaogang Chen, Pengxu Wei, Wei Ke, Qixiang Ye, and Jianbin Jiao. Pedestrian Detection with Deep Convolutional Neural Network, pages 354-365. Springer International Publishing, Cham, 2015.

[15] Jörg Wagner, Volker Fischer, Michael Herman, and Sven Behnke. Multispectral pedestrian detection using deep fusion convolutional neural networks. In 24th European Symposium on Artificial Neural Networks, Computational Intelligence and Machine Learning (ESANN), pages 509-514, April 2016.

[16] Dănuţ Ovidiu Pop, Alexandrina Rogozan, Fawzi Nashashibi, and Abdelaziz Bensrhair. Fusion of stereo vision for pedestrian recognition using convolutional neural networks. Proceedings of the European Sympoisum on Artificial Neural Networks (ESANN), 2017.

[17] David Vazquez, Antonio M. Lopez, Javier Marin, Daniel Ponsa, and David Geronimo. Virtual and real world adaptation for pedestrian detection. IEEE Transactions on Pattern Analysis \& Machine Intelligence, 36(4):797-809, 2014.

[18] C. Karaoguz and A. Gepperth. Incremental learning for bootstrapping object classifier models. In 2016 IEEE 19th International Conference on Intelligent Transportation Systems (ITSC), pages 1242-1248, Nov 2016.
[19] Yann Lecun, Léon Bottou, Yoshua Bengio, and Patrick Haffner. Gradient-based learning applied to document recognition. In Proceedings of the IEEE, pages 2278-2324, 1998.

[20] Alex Krizhevsky, Ilya Sutskever, and Geoffrey E Hinton. Imagenet classification with deep convolutional neural networks. In F. Pereira, C. J. C. Burges, L. Bottou, and K. Q. Weinberger, editors, Advances in Neural Information Processing Systems 25, pages 1097-1105. Curran Associates, Inc., 2012. 